\title{
Microbial Community Compositional Analysis for Series Reactors Treating High Level Antibiotic Wastewater
}

\author{
Yanqin Deng, Yu Zhang, Yingxin Gao, Dong Li, Ruyin Liu, Miaomiao Liu, Haifeng Zhang, Bo Hu, Tao Yu, \\ and Min Yang*
}

State Key Laboratory of Environmental Aquatic Chemistry, Research Center for Eco-Environmental Sciences, Chinese Academy of Sciences, P.O. Box 2871, Beijing 100085, China

\section{Supporting Information}

ABSTRACT: A full-scale biosystem consisting of two anaerobic reactors ( $\mathrm{HA}$ and $\mathrm{BF} 1)$ and four aerobic ones (BF2-BF4 and $\mathrm{OD}$ ) in succession and receiving antibiotic-bearing (mainly streptomycin) wastewater was used for studying the impacts of antibiotics on microbial community structures. Significant decreases of streptomycin (from $3955 \pm 1910$ to $23.1 \pm 4.7$ $\left.\mu \mathrm{g} \mathrm{L}^{-1}\right)$ and $\mathrm{COD}_{\mathrm{Cr}}$ were observed along the treatment process. Cloning results show that the anaerobic reactors (HA and BF1) were dominated with Deltaproteobacteria (51\%) mainly affiliated with sulfate-reducing bacteria (SRB), while the aerobic BF2 receiving streptomycin of $408.6 \pm 59.7 \mu \mathrm{g} \mathrm{L}^{-1}$ was dominated with Betaproteobacteria (34\%), Deltaproteobacteria (31\%) and Bacteroidetes (14\%). Gammaproteobacteria (15.9-22.4\%), Beta-

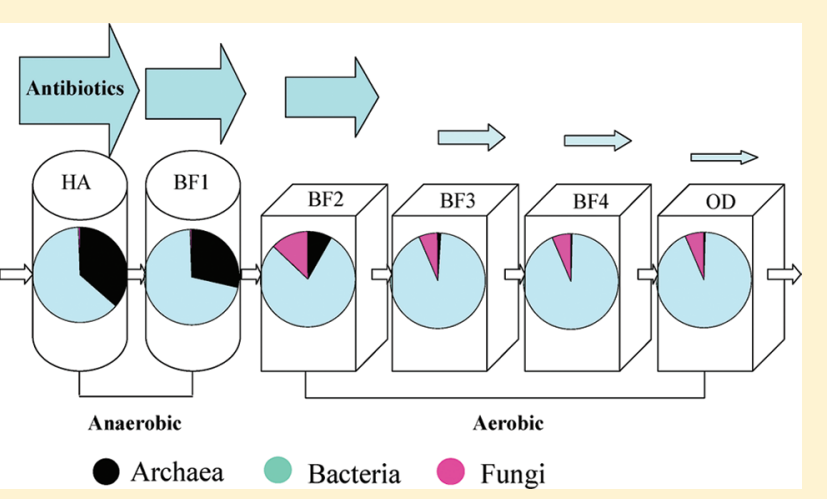
proteobacteria (10.0-20.3\%), and Bacteroidetes (4.5-29.7\%) became the major bacterial groups in aerobic BF3-OD receiving streptomycin of $\leq 83 \pm 13 \mu \mathrm{g} \mathrm{L} \mathrm{L}^{-1}$. Archaea affiliated with Methanomethylovorans hollandica-like methylotroph was abundant in $\mathrm{HA}$ and BF1 (archaea/bacteria, 0.54-0.40; based on specific gene copy number), suggesting the coexistence of SRB and methanogens in degrading pollutants. Fungi were abundant (fungi/bacteria, 0.15 ; based on specific gene copy number) with the dominance of Ascomycota (clone ratio of Ascomycota/eukarya, 25.5\%) in BF2, suggesting that fungi could be an important player in pollutant removal under high levels of antibiotics. This study demonstrates that under high antibiotic levels, wastewater treatment communities may maintain system stability through adjusting bacterial, archaeal, and eukaryal compositions.

\section{INTRODUCTION}

There has been increasing concern over the pollution of environments by antibiotics due to the fact that antibioticresistant bacteria become dominant in such ecosystems. ${ }^{1,2}$ Our previous studies have shown that antibiotic production waste streams could contain much higher concentrations of antibiotics than normal aquatic environments. ${ }^{3,4}$ Except for the problem of production of large amount of antibiotic resistant genes $^{3}$ the presence of high concentration antibiotics in wastewater could also trigger significant changes of wastewater treatment microbial communities, and might disturb the stability and performance of biological wastewater treatment systems due to the strong bacteriostatic effects of antibiotics.

Some studies have investigated the impacts of antibiotics on bacterial communities. ${ }^{5,6}$ A reduction in bacterial number together with alterations in microbial populations were observed in a model sewage purification system when antibiotics were added in concentrations $\left(2-5 \mathrm{mg} \mathrm{L}^{-1}\right)$ that may occur in hospital wastewater. ${ }^{7}$ According to our culturebased studies, ${ }^{3,4}$ several bacterial groups such as Pseudomonas species (56\%) and Stenotrophomonas maltophilia etc. (14\%) have been linked with aquatic environments containing high concentrations of oxytetracycline (OTC), while those from a wastewater treatment plant (WWTP) receiving penicillin G (PEN) were Pseudomonas species (24\%), Brachymonas denitrificans (21\%) and Psychrobacter pulmonis (20\%). However, culture-based approach may lead to the favored growth of some special bacterial groups, ${ }^{8}$ and the impacts of antibiotics on bacterial communities in full-scale WWTPs are in general not well understood.

Except for bacteria, eukarya such as fungi, which can decompose complex organic compounds in wastewater ${ }^{9,10}$ but are normally neglected, are often found in aerobic wastewater treatment systems. ${ }^{11}$ At the same time, methanogenic archaea, which can play a critical role in methane generation in the anaerobic food web by cooperating with bacteria, ${ }^{12}$ are an important group of microbes involved in anaerobic wastewater treatment. ${ }^{13}$ Since antibiotics target bacteria specifically, it is easy to assume that archaea and fungi, which interact with bacteria respectively in anaerobic and aerobic reactors, may also be influenced indirectly by antibiotics in wastewater. However,

Received: July 26, 2011

Revised: November 23, 2011

Accepted: November 30, 2011

Published: November 30, 2011 
Table 1. Variations of Antibiotics along the Treatment Process

\begin{tabular}{|c|c|c|c|c|c|c|c|c|c|}
\hline \multirow[b]{2}{*}{$\begin{array}{c}\text { antibiotics }(\mu \mathrm{g} \\
\left.\mathrm{L}^{-1}\right)\end{array}$} & & \multirow[b]{2}{*}{ influent } & \multicolumn{6}{|c|}{ effluent antibiotic concentrations of each bioreactor } & \multirow[b]{2}{*}{$\begin{array}{c}\text { removal } \\
(\text { mean \%) }\end{array}$} \\
\hline & & & HA & BF1 & BF2 & BF3 & BF4 & OD & \\
\hline aminoglycoside & streptomycin & $3955.4 \pm 1910.7$ & $1477.4 \pm 713.7$ & $408.6 \pm 59.7$ & $83.4 \pm 13.1$ & $60.1 \pm 14.9$ & $23.1 \pm 1.60$ & $23.1 \pm 4.70$ & 99.4 \\
\hline \multirow[t]{11}{*}{ beta-lactams } & cefradine & $5.22 \pm 3.91$ & $0.27 \pm 0.17$ & $0.26 \pm 0.16$ & $0.16 \pm 0.12$ & $0.16 \pm 0.14$ & $0.17 \pm 0.16$ & $0.16 \pm 0.12$ & 96.9 \\
\hline & ceftrixone & $4.90 \pm 3.79$ & $0.46 \pm 0.38$ & $0.45 \pm 0.40$ & $0.39 \pm 0.34$ & $0.45 \pm 0.38$ & $0.46 \pm 0.40$ & $0.45 \pm 0.40$ & 90.8 \\
\hline & cefataxime & $1.34 \pm 1.24$ & $0.14 \pm 0.12$ & $0.14 \pm 0.10$ & $0.11 \pm 0.08$ & $0.09 \pm 0.06$ & $0.08 \pm 0.05$ & $0.08 \pm 0.05$ & 94.0 \\
\hline & cefalexin & $0.64 \pm 0.32$ & $0.07 \pm 0.06$ & $0.03 \pm 0.01$ & $0.02 \pm 0.01$ & $0.03 \pm 0.02$ & $0.03 \pm 0.02$ & $0.03 \pm 0.02$ & 95.3 \\
\hline & PEN G & $0.34 \pm 0.19$ & $0.17 \pm 0.05$ & $0.17 \pm 0.05$ & $0.17 \pm 0.05$ & $0.17 \pm 0.05$ & $0.16 \pm 0.04$ & $0.16 \pm 0.04$ & 52.9 \\
\hline & amoxicillin & $0.50 \pm 0.46$ & $0.50 \pm 0.46$ & $0.20 \pm 0.16$ & $0.11 \pm 0.08$ & $0.08 \pm 0.05$ & $0.05 \pm 0.02$ & $0.05 \pm 0.02$ & 90.0 \\
\hline & ampicillin & $0.32 \pm 0.08$ & $0.32 \pm 0.09$ & $0.31 \pm 0.09$ & $0.27 \pm 0.05$ & $0.25 \pm 0.04$ & $0.13 \pm 0.08$ & $0.09 \pm 0.08$ & 71.9 \\
\hline & cefazolin & $0.13 \pm 0.12$ & $0.12 \pm 0.11$ & $0.12 \pm 0.11$ & $0.12 \pm 0.11$ & $0.12 \pm 0.11$ & $0.12 \pm 0.11$ & $0.12 \pm 0.11$ & 7.70 \\
\hline & cefuroxime & $0.07 \pm 0.03$ & $0.05 \pm 0.01$ & $0.04 \pm 0.01$ & $0.04 \pm 0.02$ & $0.03 \pm 0.01$ & $0.02 \pm 0.01$ & $0.02 \pm 0.01$ & 71.4 \\
\hline & cefadroxil & $0.02 \pm 0.01$ & $0.02 \pm 0.01$ & $0.01 \pm 0.01$ & $0.01 \pm 0.01$ & $0.01 \pm 0.01$ & $0.01 \pm 0.01$ & $0.01 \pm 0.01$ & 50.0 \\
\hline & total & $13.5 \pm 10.2$ & $2.1 \pm 1.5$ & $1.7 \pm 1.1$ & $1.4 \pm 0.9$ & $1.4 \pm 0.9$ & $1.2 \pm 0.9$ & $1.2 \pm 0.9$ & 91.3 \\
\hline
\end{tabular}

little information is available regarding the impact of residual antibiotics on the relationship between bacterial and archaeal/ eukaryal communities under high antibiotic levels.

In this study, the impacts of antibiotic residues on wastewater treatment communities were revealed by investigating changes of microbial community structures as well as antibiotic concentrations along a WWTP consisting of an anaerobic hydrolysis (HA) reactor, four biological contact reactors (BF1$\mathrm{BF} 4$ ), and an oxidation ditch (OD) reactor, which receives wastewater containing eleven antibiotics with streptomycin, an aminoglycoside bactericide, as the dominant one. Antibiotics were determined using liquid chromatography-tandem mass spectrometry (LC/MS/MS), and the bacterial, eukaryal, and archaeal community structures of each reactor were characterized using multiple techniques including real-time PCR, denaturing gradient gel electrophoresis (DGGE) and clone library construction. The purpose of this study was to provide useful information for optimizing the design and operation of WWTPs receiving antibiotic-bearing wastewater. This study will help understand the characteristics of wastewater treatment microbial communities, particularly the relationship between bacterial and archaeal/eukaryal communities under high antibiotic levels.

\section{MATERIALS AND METHODS}

Description of WWTP and Sample Collection. Liangcun WWTP, located in Shijiazhuang City, China, receives a mixture of pretreated pharmaceutical effluents $(70-80 \%)$ and municipal wastewater with an average flow of $50000 \mathrm{~m}^{3}$ day $^{-1}$. As shown in SI Figure S1, the WWTP consists of a sludge-bed type hydrolysis tank (HA), four biological contact oxidation tanks (BF1-BF4) filled with columnar type plastic biocarriers $(\Phi 10 \times 10-20 \mathrm{~mm})$, and an oxidation ditch (OD) (mixed liquor suspended solids, 3-4 $\mathrm{g} \mathrm{L}^{-1}$ ) in succession. HA and BF1 were under anaerobic condition, while BF2-OD were under aerobic condition. Wastewater sampling was conducted once a week during August and September, 2009 (five times in total). All wastewater samples were taken as flow-proportional composite samples using automated samplers that collected defined volumes every hour over a $24 \mathrm{~h}$ period, and were stored on ice for transport back to the laboratory. Concurrently, sludge samples were collected from each reactor. Detailed description of the WWTP is given in the Supporting Information (SI).
Analytical Methods. Wastewater parameters such as $\mathrm{COD}_{\mathrm{Cr}}$ were determined in accordance with Standard Methods. ${ }^{14}$ Residual antibiotics including streptomycin and 10 beta-lactams were analyzed using LC/MS/MS, ${ }^{15,16}$ and the analytical methods are detailed in the SI. All samples were analyzed in triplicate.

DGGE Analysis. Before PCR amplification, total DNA from the sludge samples was extracted using the FastDNA SPIN Kit for Soil (Qbiogene, Carlsbad, CA). The DNA concentrations were determined in triplicate using a spectrophotometer (Nanodrop Technologies, Rockland, DE). The DNA products were then applied for DGGE and other molecular analyses. Primers GC-EUB341f/EUB534r and NS1/GC-fung were, respectively, used for bacterial and eukaryal analyses. For archaea, two-step nested DGGE was used: the PCR products obtained using primers Arch21f/Arch958r were then used as a template for a second amplification with primers GC-arch344f/ Arch519r. Detailed information for the primers are included in SI Table S2, and procedures for PCR amplification, DGGE, and cluster analysis of DGGE dendrograms are detailed in the SI.

Real-Time PCR Assays. Real-time PCR assays were performed for the quantification of bacterial 16S rRNA, archaeal 16S rRNA and fungal rRNA genes using primers 338f/518r, 931f/M1100r and ITS1/ITS4, respectively (SI Table S2). Thermal cycling conditions consisted of $30 \mathrm{~s}$ at 95 ${ }^{\circ} \mathrm{C}$ followed by 40 amplification cycles of $10 \mathrm{~s}$ at $95{ }^{\circ} \mathrm{C}, 15 \mathrm{~s}$ at an annealing temperature $\left(60{ }^{\circ} \mathrm{C}\right.$ for bacterial and archaeal genes, and $55{ }^{\circ} \mathrm{C}$ for fungal gene), and $15 \mathrm{~s}$ at $72{ }^{\circ} \mathrm{C}$. A melt curve profile was obtained by heating the mixture to $95{ }^{\circ} \mathrm{C}$, cooling to $65{ }^{\circ} \mathrm{C}(15 \mathrm{~s})$, and slowly heating to 95 at $0.1{ }^{\circ} \mathrm{C} \mathrm{s}^{-1}$ with continuous measurement of fluorescence. PCR quality water was used as negative control. All samples were run in triplicate. A duplicate 10-fold dilution series of standard DNA was used to generate a standard curve. The standard curves for bacterial, archaeal and fungal genes had $R^{2}$ values of $0.95-0.99$ and the amplification efficiencies were 90-110\%.

Construction of Bacterial, Archaeal and Eukaryal Gene Clone Libraries. Bacterial, archaeal, and eukaryal gene clone libraries were constructed as detailed in the SI. Primers 27f/1492r, Arch21f/Arch958r, NS1/NS2 (SI Table S2) were used to amplify fragments of ca. $1500 \mathrm{bp}$ bacterial $16 \mathrm{~S}$ rRNA, $950 \mathrm{bp}$ archaeal $16 \mathrm{~S}$ rRNA, and $550 \mathrm{bp}$ eukaryal $18 \mathrm{~S}$ rRNA genes, respectively. The PCR products were then used for cloning, in accordance with the manufacturer's protocol (TA cloning kit, Invitrogen). Positive clones were sequenced with an ABI 3730 automated sequencer (Invitrogen, Shanghai). The 
obtained sequences were compiled and compared to available rDNA sequences in GenBank using the NCBI BLAST program.

Statistical Analysis. Sørensen's similarity index ${ }^{17}$ was used to compute bacterial community similarity of each treatment unit using PrimerE software v. Six. ${ }^{18}$ Multidimensional scaling (MDS) plots were constructed to arrange samples in twodimensional space according to their relative similarities. Mantel tests were performed using R 2.9.1 (http://www.rproject.org/) to evaluate correlation between antibiotic concentrations, $\mathrm{COD}_{\mathrm{Cr}}$, bacterial and eukaryal community structures (revealed by phylogenetic analysis) in the four aerobic bioreactors (BF2-OD). The other statistical analyses were all performed by using the SPSS version 16.0 release.

Nucleotide Sequence Accession Numbers. The nucleotide sequence data reported in this paper have been deposited in the NCBI (GenBank) database under accession numbers HQ440107 to HQ440122 for archaeal clones and DGGE bands; HQ440123 to HQ440131 for eukaryal DGGE bands; HQ440040 to HQ440090 and HM584299 to HM584364 for bacterial clones; and JN120055 to JN120086 for eukaryal clones.

\section{RESULTS}

Treatment Performance of the System. Table 1 shows the changes in concentrations of antibiotics along the treatment process. A total of eleven antibiotics belonging to two classes were detected in the WWTP influent with streptomycin as the dominant one (Table 1). The removals of streptomycin and beta-lactams by the system were respectively over $99 \%$ and $91 \%$, with most of beta-lactams (85.4\%) removed in HA. $\mathrm{COD}_{\mathrm{Cr}}$ of the influent and the final effluent, respectively, valued $475 \pm 25 \mathrm{mg} \mathrm{L}^{-1}$ and $120 \pm 20 \mathrm{mg} \mathrm{L}^{-1}$. While $30-35$ $\mathrm{mg} \mathrm{L}^{-1}$ of $\mathrm{COD}_{\mathrm{Cr}}$ was removed in the anaerobic $\mathrm{HA}$ and $\mathrm{BF} 1$, higher $\mathrm{COD}_{\mathrm{Cr}}$ removals $\left(60-100 \mathrm{mg} \mathrm{L}^{-1}\right)$ were achieved in the aerobic reactors (BF2-OD). Overall treatment parameters and performance are shown in SI Table S1.

Quantification of Different Microbial Groups by RealTime PCR. As Figure 1 shows, the copy ratios of archaea/

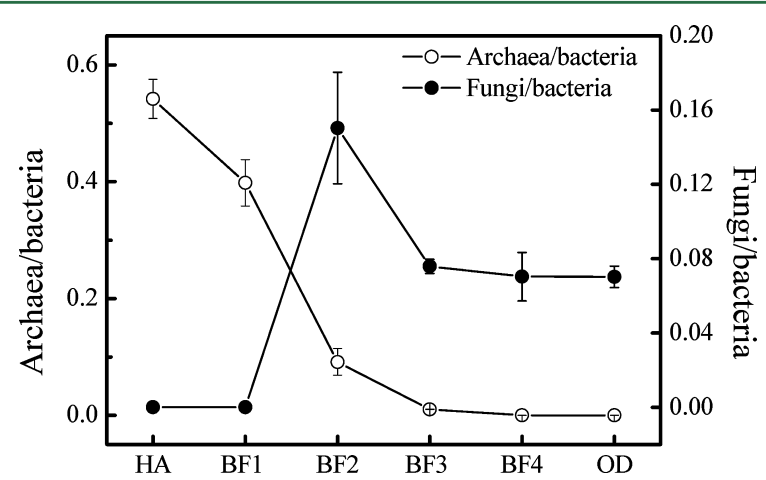

Figure 1. Ratios of the eukarya/bacteria as well as archaea/bacteria in each reactor revealed by real-time PCR.

bacteria were very high $(0.54-0.40)$ in HA and BF1, but were very low in the following reactors $(<0.10)$. The copy ratios of fungi/bacteria were negligible $\left(3.25 \times 10^{-5}-2.75 \times 10^{-5}\right)$ in $\mathrm{HA}$ and $\mathrm{BF} 1$, and increased to the maximum value of 0.15 in $\mathrm{BF} 2$, then decreased to 0.070-0.075 in BF3-OD.

Compositions of Different Microbial Groups. Changes in archaeal, bacterial and eukaryal compositions along the process were revealed using PCR-DGGE, and totally six bacterial, two archaeal (HA and BF1), and four eukaryal (BF2OD) clone libraries.

Bacterial Compositions. UPGMA (Unweighted Pair Group Method with Arithmetic Mean) analysis (SI Figure S3) shows that the bacterial communities of HA and BF1 were clustered together with high similarity (Jaccard's coefficient, $94 \%)$, while those of $\mathrm{BF} 3$ and $\mathrm{BF} 4$ were clustered together (84\%). BF2 was closer to the HA/BF1 group (80\%), but OD was quite different from all of the other groups (67\%). Sequencing results show that HA and BF1 were dominated by SRB (B1, Desulfocapsa thiozymogenes; B3, uncultured Desulforhopalus species; B7, Desulfobacterium aniline), while other species (B2, Bdellovibrio species; B4, Frigoribacterium species; $\mathrm{B} 8$, Chloroflexi bacterium) became the major groups in BF3BF4 (Table S4). BF2 shared some common bands with HA and BF1 (B1, D. thiozymogenes; B7, D. anilini), and other common bands with BF3 and BF4 (B5, uncultured Betaproteobacterium clone; B6, Syntrophaceae bacterium; B4, Frigoribacterium species). Some bands were specific to BF3, BF4, and OD (B2, Bdellovibrio species).

For bacterial clone libraries, $33516 \mathrm{~S}$ rDNA clones were selected and grouped into 97 OTUs (SI Table S5). As shown in Figure 2 and SI Table S5, both HA and BF1 were dominated

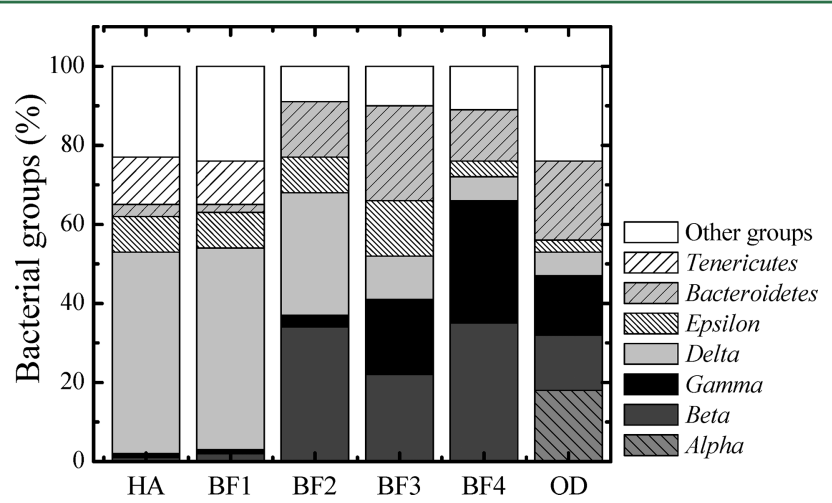

Figure 2. Percentages of the bacterial groups in the six reactors (Alpha, Alphaproteobacteria: Beta, Betaproteobacteria; Gamma, Gammaproteobacteria; Delta, Deltaproteobacteria; Epsilon, Epsilonproteobacteria).

with Deltaproteobacteria (51\%), followed by Tenericutes (1112\%) and Epsilonproteobacteria (9\%), while BF2 was dominated with Betaproteobacteria (34\%) and Deltaproteobacteria (31\%), followed by Bacteroidetes (14\%) and Epsilonproteobacteria (9\%). In comparison, Gammaproteobacteria (15.9-22.4\%), Betaproteobacteria (10.0-20.3\%), and Bacteroidetes (4.5-29.7\%) became the major bacterial groups with an even distribution in BF3-OD. Alphaproteobacteria (17.3\%) was only detected in OD.

The MDS procedure resulted in a two-dimensional bacterial profile in each site according to clone library results (SI Figure S4). The bacterial communities could be clustered into four groups: HA-BF1, BF2, BF3-BF4, and OD, which was in accordance with the UPGMA analysis of DGGE result. Mantel test shows that the changes of bacterial community structures were significantly related with the concentrations of residual antibiotics (streptomycin and beta-lactams) $(p=0.045)$ and have marginally significant correlation with $\operatorname{COD}_{\mathrm{Cr}}(p=0.079)$. (Table 2).

Archaeal Compositions. Similarly, UPGMA analysis (SI Figure S3) shows that $\mathrm{HA}$ and BF1 were clustered together 
Table 2. Analysis of Correlation between Bacterial Community Structures in the Four Aerobic Reactors and Several Environmental Factors

\begin{tabular}{|c|c|c|}
\hline \multirow[b]{2}{*}{ variable } & \multicolumn{2}{|c|}{ bacterial community $^{b}$} \\
\hline & $R$-value & $P$-value \\
\hline streptomycin & 0.749 & 0.091 \\
\hline beta-lactams & 0.827 & 0.081 \\
\hline total antibiotics & 0.809 & $0.045^{a}$ \\
\hline $\mathrm{COD}_{\mathrm{Cr}}$ & 0.659 & 0.079 \\
\hline
\end{tabular}

${ }^{a}$ Significant correlation $(p<0.05) .{ }^{b}$ Percentage of individual phylum clones in each bacterial clone library.

with high similarity (Jaccard's coefficient, 93\%), whereas BF3 and BF4 were clustered together (80\%). Sequencing results show that most of the bands belonged to methanogenic archaea (Ar1-Ar11), including Methanomethylovorans hollandica, uncultured Methanosaeta species, uncultured Methanosarcinales archaeon, etc. (SI Table S4).

Archaeal clone libraries were constructed for samples from $\mathrm{HA}$ and BF1. In total, 105 positive clones were selected and grouped into four OTUs, showing a very low diversity of archaea in the system. Methanomethylovorans hollandica was dominant (84.6-90.6\%), with uncultured crenarchaeote clone (5.7-7.7\%), M. mazeii (1.9-3.8\%), and M. victoriae (1.9$3.8 \%)$ in low abundance.

Eukaryal Compositions. The DGGE profiles of $18 \mathrm{~S}$ rRNA genes from HA and BF1 were clustered together (Jaccard's coefficient, $78 \%$ ), and those of BF3 and BF4 were clustered together (60\%) (SI Figure S3). BF4 and OD showed quite different band patterns from each other and from BF2 and BF3. Among the retrieved 18S rRNA gene sequences, eight belonged to fungi and three belonged to Protista (respectively affiliated with Choanoflagellida, Alveolata, and Rhizaria; SI Table S4). Although the primer set NS1 and GC-fung was originally designed for fungi (SI Table S2), eukaryal genes outside fungi were also detected.

Four eukaryal clone libraries were constructed for samples from the four aerobic reactors (BF2-OD). In total, 190 positive clones were acquired and grouped into 27 eukaryal OTUs, belonging to fungi (5 OTUs, including Ascomycota, Blastocladiomycota, and unclassified fungi clones), Alveolata (6 OTUs), Metazoa (2 OTUs), Rhizaria (1 OTU, affiliated with Cercozoa), Ichthyosporea (3 OTUs), Amoebozoa (1 OTU), Trimastix (1 OTU), and unclassified (8 OTUs) (detailed in SI Table S6). The MDS analysis based on clone library results shows that the eukaryal community structures were clustered into three groups: BF2, BF3/BF4 and OD (SI Figure S4).

The distribution of the eukaryal groups in the four aerobic reactors is shown in Figure 3 and SI Table S6. Besides Alveolata (48.9\%), fungi affiliated with Ascomycota were dominant in BF2 (25.5\%). However, fungi were not detected in BF3 and BF4, although real-time PCR analysis shows that the copy ratios of fungi/bacteria were $0.07-0.075$. Both $\mathrm{BF} 3$ and $\mathrm{BF} 4$ were mainly dominated with Alveolata (100\% in BF3 and $91.2 \%$ in BF4). The Alveolata in BF2-BF4 were mainly affiliated with heterotrophic Ciliophora (38.3-90.9\%). On the other hand, OD shows a more diverse eukaryal composition with Alveolata (3.8\%), fungi (11.3\%; including Ascomycota, 3.9\%), Metazoa (20.4\%), Rhizaria (14.8\%), Ichthyosporea (5.6\%) and many unclassified clones (42.7\%). The eukaryal community structures showed marginally significant relation with the bacterial

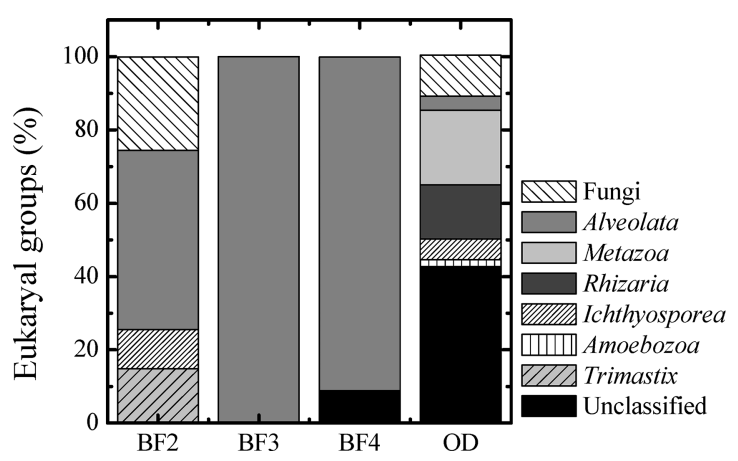

Figure 3. Percentages of the eukaryal groups in the four aerobic reactors.

community structures $(r=0.899, p=0.068)$, but no significant relation with antibiotics $(p=0.357)$ and $\operatorname{COD}_{\mathrm{Cr}}(p=0.385)$.

\section{DISCUSSION}

There are only several reports available showing the impacts of antibiotics on microbial communities and their functions during wastewater treatment. Based on simulation experiments, Halling-Sorensen observed the inhibitory effects of tetracyclines on aerobic growth and nitrification of activated sludge. ${ }^{6}$ However, few studies have focused on long-term impacts of antibiotics on the relationship between bacteria and other major microbial groups in wastewater systems.

In this study, a full-scale multiple-step biosystem including two anaerobic reactors and four aerobic reactors was used for studying the impacts of residual antibiotics on microbial community structures. The two anaerobic reactors exhibited high removals of both streptomycin $(89.7 \%)$ and beta-lactam antibiotics $(88.3 \%)$ (Table 1), which accords with previous reports that ampicillin and aureomycin can be partially degraded in an anaerobic reactor. ${ }^{19}$ The influent streptomycin concentrations in HA and BF1 (3955.4 \pm 1910.7 and 1477.4 \pm $713.7 \mu \mathrm{g} \mathrm{L}^{-1}$, respectively) as well in aerobic BF2 (408.6 \pm 59.7 $\left.\mu \mathrm{g} \mathrm{L}^{-1}\right)$ were very high in comparison with those in the succeeding aerobic reactors $\left(83 \pm 13\right.$ to $\left.23 \pm 1.6 \mu \mathrm{g} \mathrm{L}^{-1}\right)$. The distribution of streptomycin concentrations along the treatment process provide an in situ environment to study how the microbial community structures were influenced by different levels of residual antibiotics. The total beta-lactams concentrations $\left(1.2-1.4 \mu \mathrm{g} \mathrm{L}^{-1}\right)$ in the aerobic reactors were much lower than minimum inhibitory concentrations (MICs) of sensitive strains (the MICs of PEN for sensitive strains, 15-31 $\left.\mu \mathrm{g} \mathrm{L}^{-1}\right),{ }^{20}$ but were still much higher than those found in surface waters or even municipal wastewater. ${ }^{16}$

Impacts of Antibiotics on Bacterial Community Structures. The Gram-positive Firmicutes are generally major bacterial constituents in anaerobic bioreactors. ${ }^{21,22}$ The physiological types like Acetobacterium belonging to the large phylogenetic group of Gram-positive clostridia in Firmicutes play an important role in producing methanogenic precursors such as acetic acid during anaerobic digestion. ${ }^{23}$ In this study, however, no Firmicutes but Gram-negative bacteria such as SRB in Deltaproteobacteria (51\%) and Acholeplasma species in Epsilonproteobacteria (9\%) were dominant in HA/BF1. This was significantly different from the anaerobic reactor of a system for treating inosin producing (by fermentation) wastewater, which contained abundant Firmicutes (59.6\%) but no SRB (SI Table S7). Owing to their ubiquitous distribution in the environment, SRB have an important role in the sulfur 
and carbon cycles and can adapt to various extreme environments. $^{24}$ It has been reported that some SRB are resistant to many kinds of broad-spectrum antibiotics including metronidazole, ciprofloxacin and sulfasalazine. ${ }^{25,26}$ So, it is assumed that the presence of high concentrations of streptomycin may have favored the growth of SRB.

Bacterial community structures in the four aerobic reactors were further compared (Figure 2). The Mantel test results revealed that the concentrations of antibiotics (streptomycin and total beta-lactams) were significantly $(p=0.045)$ correlated to bacterial compositions in the four aerobic reactors (Table 2), showing that the antibiotics may have played an important role in shaping bacterial community structures. A sensitive bacterial strain has exhibited growth inhibition to streptomycin at a concentration of $190 \mu \mathrm{g} \mathrm{L}{ }^{-1} .^{27}$ With an influent streptomycin concentration of $408.6 \pm 59.7 \mu \mathrm{g} \mathrm{L}^{-1}$, it is possible that bacteria in BF2 were still under high antibiotic level. In BF2, Betaproteobacteria (affiliated mainly with M. universalis and D. oestradiolicum) and Bacteroidetes (affiliated with uncultured Saprospiraceae bacterium), which exist normally in aerobic biosystems, became abundant (34\% and $14 \%$, respectively) (Figure 2 and SI Table S5). Betaproteobacteria have been considered as the original source of Class 1 integrons, which can capture and express diverse resistance genes and are central players in the worldwide problem of antibiotic resistance. ${ }^{28}$ Our previous study ${ }^{4}$ has found that abundant Class 1 integrons, which contains aminoglycoside resistance genes (aadA1, aadA2, and aadA2a) as one of the major resistant genes, existed in OTC production wastewater. Some Bacteroides species harbor both conjugative plasmids and transposons, and possess resistance to erythromycin and tetracycline. ${ }^{29}$ Considering the relatively high influent antibiotic concentration in BF2 (408.6 \pm $\left.59.7 \mu \mathrm{g} \mathrm{L}^{-1}\right)$, it is possible that the significantly increased abundance of Betaproteobacteria and Bacteroides were related with their ability to acquire antibiotic resistance genes. Bacteria from Betaproteobacteria and Bacteroides could degrade a variety of pollutants, and play important roles in wastewater treatment. $^{30,31}$

Gammaproteobacteria exist normally in aerobic biosystems as the main bacterial groups. ${ }^{32-34}$ Compared to the very low abundance of Gammaproteobacteria (clone ratio, 3\%) in BF2, the abundance of Gammaproteobacteria increased significantly in BF3-OD (15-31\%), which might be related to the much lower level of influent antibiotics $(23.1 \pm 1.60-83.4 \pm 13.1 \mu \mathrm{g}$ $\mathrm{L}^{-1}$ ) (Table 1, Figure 2). Alphaproteobacteria (18.0\%) clones related to uncultured Caulobacterales bacterium and Sphingomonas species as well as some minor bacterial groups only occurred in OD (Table 1, Figure 2, SI Table S5). In general, the bacterial community compositions in BF3-OD exhibited more similar characteristics typically observed for aerobic wastewater treatment systems, where the microbial communities are usually dominated by Betaproteobacteria, Gammaproteobacteria and (or) Alphaproteobacteria (41-66\% in total, by $16 \mathrm{~S}$ rDNA analysis), ${ }^{32-34}$ showing that the impacts of residual antibiotics might become less profound under the relatively low antibiotic levels. It seemed that the Gammaproteobacteria, Alphaproteobacteria and some other bacterial populations were more sensitive to streptomycin as a broad-spectrum bactericidal antibiotic, than Betaproteobacteria and Bacteroidetes. The result is different from the reports that many genera of Gammaproteobacteria including Acinetobacter, Pseudomonas and Stenotrophomonas are resistant to high concentrations of streptomycin or OTC using pure cultures, ${ }^{4,35,36}$ which might be due to the fact that the Gammaproteobacteria isolates are easier to be acquired using culture methods.

Archaeal Community Structures and Their Relation with Bacterial Groups in Two Anaerobic Reactors. Archaea were the dominant microbial group in $\mathrm{HA}$ and $\mathrm{BF} 1$ (archaea/bacteria, 0.54-0.40) (Figure 1), with the Methanomethylovorans hollandica-like methylotroph as the dominant species (87-97\%). This species is a key consumer of dimethyl sulfide and methanethiol, as well as methanol and methylamines. ${ }^{22}$ Both the methanogenic archaea and SRB catalyze the terminal stages of the anaerobic mineralization of organic compounds produced by acetogenic bacteria from complex organics, ${ }^{12,37}$ with SRB often outcompeting methanogens. ${ }^{12} \mathrm{On}$ the other hand, a large number of SRB have the ability to grow on fatty acids and aromatic compounds, and can degrade organic compounds incompletely to small molecular volatile acids. $^{24}$ The coexistence of methanogens with SRB in HA/BF1 without the perceptible existence of Gram-positive acetogenic bacteria suggests that $\mathrm{SRB}$ in $\mathrm{HA}$ and $\mathrm{BF} 1$ may be playing an important role in the breakdown of complex organic matter for the methanogens. ${ }^{38}$

Eukaryal Community Structures and Their Relation with Bacterial Groups in Four Aerobic Reactors. The existence of some antibiotics have significantly resulted in the dose related shifts in the fungal: bacterial ratio in soil ${ }^{39}$ or aquatic media. ${ }^{40}$ Real-time PCR analyses show that the fungi/ bacteria was decreasing along the four aerobic reactors (from 0.15 in BF2 to 0.01 in OD), but were still higher than those in sewage treatment plants acquired by culturing method (fungi/ bacteria, $\left.7.0 \times 10^{-4}-0.002\right) .{ }^{41}$ The fungi/bacteria ratio of activated sludge from an inosine-producing WWTP was determined by real-time PCR for comparison, and exhibited an extremely low fungi/bacteria ratio $\left(1.52 \times 10^{-6}\right)$. Though further study is required, it seemed that fungi normally exist at low abundance in conventional WWTPs. So the above results show that the relatively high residual antibiotic concentration in BF2 might have favored the growth of fungi, particularly Ascomycota, as will be discussed below.

Phylogenetic analysis confirmed that much more Ascomycota existed in BF2 (25.5\%) than the other aerobic reactors (0$3.8 \%)$. The Ascomycota/eukarya shows a decreasing trend along the four aerobic reactors. Ascomycota can decompose complex organic compounds such as PAHs, oil and phenol in wastewater. ${ }^{9,10}$ So the high abundance of Ascomycota in BF2 suggests that fungi may have contributed much to pollutant degradation during wastewater treatment since the aerobic functional populations including Gammaproteobacteria (3\%) and Alphaproteobacteria (0\%) were inhibited. Mantel test (Table 2) shows that the eukaryal community structures in the four aerobic reactors have marginally significant correlation with bacterial community structures $(p=0.068)$. As the presence of the antibiotics has significant impacts on bacterial community structures, it may also affect the eukaryal community structures indirectly. It might be possible that fungi have some sort of association with bacteria mainly from Betaproteobacteria and Bacteroides in pollutant removal in BF2. By including abundant eukarya into wastewater treatment microbial communities, wastewater treatment systems could maintain a relatively good stability and performance even when some important functional bacteria are lost. Further studies are required to explore the possible functions of the major eukaryal groups. 
Abundant Alveolata, which are mainly affiliated with the heterogeneous Ciliophora, were identified in BF2-BF4 (48.8$100 \%$ ) (Figure 3, SI Table S6). These eukaryal groups are supposed to live on predating smaller microbes like bacteria. ${ }^{42}$ The appearance of Metazoa (20.4\%), a top predator in the food chain of microbial communities in wastewater treatment, suggests that a relatively complete food chain has been established in OD. The occurrence of eukaryotes like protozoa and metazoan in municipal WWTPs has been well documented. However, information regarding their presence in industrial wastewater, particularly in antibiotic-bearing wastewater, has not been very limited. The implications of abundant Alveolata and some other eukaryotes in antibioticbearing wastewater treatment systems require further study.

\section{ASSOCIATED CONTENT}

\section{S Supporting Information}

Additional material as noted in the text. This material is available free of charge via the Internet at http://pubs.acs.org.

\section{AUTHOR INFORMATION}

\section{Corresponding Author}

* Phone: +86-6292 3475; fax: +86-6292 3541; e-mail: yangmin@rcees.ac.cn.

\section{ACKNOWLEDGMENTS}

This study was supported by National Nature Scientific Foundation of China (NSFC- 51178449, 50921064, and 20877085) and by Tianjin Key Laboratory of Aquatic Science and Technology.

\section{REFERENCES}

(1) Wittwer, M.; Keller, J.; Wassenaar, M.; Stephan, R.; Howald, D.; Rugula, G.; Bissig-Choisat, B. Genetic diversity and antibiotic resistance patterns in a Campylobacter population isolated from poultry farms in Switzerland. Appl. Environ. Microbiol. 2005, 71 (6), 2840-2847.

(2) Jindal, A.; Kocherginskaya, S.; Mehboob, A. R. M.; Mackie, R. I.; Raskin, L.; Zilles, J. L. Antimicrobial use and resistance in swine waste treatment systems. Appl. Environ. Microbiol. 2006, 72 (12), 78137820.

(3) Li., D.; Yang, M.; Hu, J. Y.; Zhang, J.; Liu, R. Y.; Gu, X.; Zhang, Y.; Wang, Z. Y. Antibiotic-resistance profile in environmental bacteria isolated from penicillin production wastewater treatment plant and the receiving river. Environ. Microbiol. 2009, 11 (6), 1506-1517.

(4) Li., D.; Yu, T.; Zhang, Y.; Yang, M.; Li, Z.; Liu, M.; Qi, R. Antibiotic resistance characteristics of environmental bacteria from an oxytetracycline production wastewater treatment plant and the receiving river. Appl. Environ. Microbiol. 2010, 76 (11), 3444-3451.

(5) Gutiérrez, I.; Watanabe, N.; Harter, T.; Glaser, B.; Radke, M. Effect of sulfonamide antibiotics on microbial diversity and activity in a Californian Mollic Haploxeralf. J. Soils Sediments 2010, 10 (3), 537544.

(6) Halling-Sorensen, B. Inhibition of aerobic growth and nitrification of bacteria in sewage sludge by antibacterial agents. Arch. Environ. Contam. Toxicol. 2002, 40 (4), 451-460.

(7) Kümmerer, K. Resistance in the environment. J. Antimicrob. Chemother. 2004, 54 (2), 311-320.

(8) Bramucci, M.; Kane, H.; Chen, M.; Nagarajan, V. Bacterial diversity in an industrial wastewater bioreactor. Appl. Microbiol. Biotechnol. 2003, 62 (5-6), 594-600.

(9) Bankar, A. V.; Kumar, A. R.; Zinjarde, S. S. Environmental and industrial applications of Yarrowia lipolytica. Appl. Microbiol. Biotechnol. 2009, 84 (5), 847-865.
(10) Deng, Y. Q.; Zhang, Y.; Hesham, A. L.; Liu, R.; Yang, M. Cell surface properties of five polycyclic aromatic compound-degrading yeast strains. Appl. Microbiol. Biotechnol. 2010, 86 (6), 1933-1939.

(11) Weber, S. D.; Ludwig, W.; Schleifer, K. H.; Fried, J. Microbial composition and structure of aerobic granular sewage biofilms. Appl. Environ. Microbiol. 2007, 73 (19), 6233-6240.

(12) Raskin, L.; Rittmann, B. E.; Stahl, D. A. Competition and coexistence of sulfate-reducing and methanogenic populations in anaerobic biofilms. Appl. Environ. Microbiol. 1996, 62 (10), 38473857.

(13) Tabatabaei, M.; Rahim, R. A.; Abdullah, N.; Wright, A. D. G.; Shirai, Y.; Sakai, K.; Sulaiman, A.; Hassan, M. A. Importance of the methanogenic archaea populations in anaerobic wastewater treatments. Process. Biochem. 2010, 45 (8), 1214-1225.

(14) APHA. Standards Methods for the Examination of Water and Wastewater, 20th ed.; American Public Health Association: Washington, DC, 1998.

(15) Cha, J. M.; Yang, S.; Carlson, K. H. Trace determination of $\beta$ lactam antibiotics in surface water and urban wastewater using liquid chromatography combined with electrospray tandem mass spectrometry. J. Chromatogr., A 2006, 1115 (1-2), 546-557.

(16) van Bruijnsvoort, M.; Ottink, S. J.; Jonker, K. M.; de Boer, E. Determination of streptomycin and dihydrostreptomycin in milk and honey by liquid chromatography with tandem mass spectrometry. $J$ .Chromatogr. A. 2004, 1058 (1-2), 137-142.

(17) Sørensen, T. A method of establishing groups of equal amplitude in plant sociology based on similarity of species and its application to analyses of the vegetation on Danish commons. K. Dan. Vidensk. Selsk. Biol. Skr. 1948, 5 (4), 1-34.

(18) Clarke, K. R.; Gorley, R. N. PRIMER v6: Users Manual/Tutorial PRIMER-E; Plymouth England, 2006.

(19) Zhou, P.; Su, C. Y.; Li, B.; Qian, Y. Treatment of high-strength pharmaceutical wastewater and removal of antibiotics in anaerobic and aerobic biological treatment processes. J. Envirin. Eng. 2006, 132 (1), $1343-1356$

(20) Yong, H.; Moyes, A.; Hood, A. Penicillin susceptibility testing of penicillinase producing Neisseria gonorrhoeae by the $\mathrm{E}$ test: a need for caution. J. Antimicrob. Chemother. 1994, 34 (4), 585-588.

(21) Narihiro, T.; Terada, T.; Kikuchi., K.; Iguchi, A.; Ikeda, M.; Yamauchi, T.; Shiraishi, K.; Kamagata, Y.; Nakamura, K.; Sekiguchi, Y. Comparative analysis of bacterial and archaeal communities in methanogenic sludge granules from upflow anaerobic sludge blanket reactors treating various food-processing, high-strength organic wastewaters. Microb. Environ. 2009, 24 (2), 88-96.

(22) Plumb, J. J.; Bell, J.; Stuckey, D. C. Microbial populations associated with treatment of an industrial dye effluent in an anaerobic baffled reactor. Appl. Environ. Microbiol. 2001, 67, 3226-3235.

(23) Collins, M. D.; Lawson, P. A.; Willems, A.; Cordoba, J. J.; Fernandez-Garayzabal, J.; Garcia, P.; Cai, J.; Hippe, H.; Farrow, J. A. E. The phylogeny of the genus Clostridium: proposal of five new genera and eleven new species combinations. Int. J. Syst. Bacteriol. 1994, 44 (4), 812-826.

(24) Muyzer, G.; Stams, A. J. The ecology and biotechnology of sulphate-reducing bacteria. Nat. Rev. Microbiol. 2008, 6, 441-454.

(25) Ohge, H.; Furne, J. K.; Springfield, J.; Sueda, T.; Madoff, R. D.; Levitt, M. D. The effect of antibiotics and bismuth on fecal hydrogen sulfide and sulfate-reducing bacteria in the rat. FEMS. Microbiol. Lett. 2003, 228 (1), 137-142.

(26) Cordova-Kreylos, A. L.; Scow, K. M. Effects of ciprofloxacin on salt marsh sediment microbial communities. ISME. J. 2007, 1 (7), 585-595.

(27) Nalecz-Jawecki, G.; Wadhia, K.; Adomas, B.; Piotrowicz-Cieslak, A. I.; Sawicki, J. Application of microbial assay for risk assessment biotest in evaluation of toxicity of human and veterinary antibiotics. Environ. Toxicol. 2010, 25 (5), 487-494.

(28) Gillings, M.; Boucher, Y.; Labbate, M.; Holmes, A.; Krishnan, S.; Holley, M.; Stokes, H. W. The evolution of class 1 Integrons and the rise of antibiotic resistance. J. Bacteriol. 2008, 190 (14), 5095-5100. 
(29) Lofmark, S.; Jernberg, C.; Jansson, J. K.; Edlund, C. Clindamycin-induced enrichment and long-term persistence of resistant Bacteroides spp. and resistance genes. J. Antimicrob. Chemother. 2006, 58 (6), 1160-1167.

(30) Wagner, M.; Loy, A. Bacterial community composition and function in sewage treatment systems. Curr. Opin. Chem. Biol. 2002, 13, 218-227.

(31) Dabert, P.; Sialve, B.; Delgenes, J. P.; Moletta, R.; Godon, J. J. Characterisation of the microbial $16 \mathrm{~S}$ rDNA diversity of an aerobic phosphorus-removal ecosystem and monitoring of its transition to nitrate respiration. Appl. Microbiol. Biotechnol. 2001, 55, 500-509.

(32) Lee, N.; Nielsen, P. H.; Aspegren, H.; Henze, M.; Schleifer, K. H.; Jansen, J. C. Long-term population dynamics and in situ physiology in activated sludge systems with enhanced biological phosphorus removal operated with and without nitrogen removal. System. Appl. Microbiol. 2003, 26 (2), 211-227.

(33) Wong, M. T.; Mino, T.; Seviour, R. J.; Onuki, M.; Liu, W. T. In situ identification and characterization of the microbial community structure of full-scale enhanced biological phosphorous removal plants in Japan. Water. Res. 2005, 39 (13), 2901-2914.

(34) Xia, S. Q.; Duan, L.; Song, Y.; Li, J.; Piceno, Y. M.; Andersen, G. L.; Alvarez-Cohen, L.; Moreno-Andrade, I.; Huang, C. L.; Hermanowicz, S. W. Bacterial community structure in geographically distributed biological wastewater treatment reactors. Environ. Sci. Technol. 2010, 44 (19), 7391-7396.

(35) Messi, P.; Guerrieri, E.; Bondi, M. Antibiotic resistance and antibacterial activity in heterotrophic bacteria of mineral water origin. Sci. Total Environ. 2005, 346 (1-3), 213-219.

(36) Mindlin, S. Z.; Soina, V. S.; Petrova, M. A.; Gorlenko, M. Z. Isolation of antibiotic resistance bacterial strains from Eastern Siberia permafrost sediments. Russ. J. Genet. 2008, 44 (1), 27-34.

(37) Dar, S. A.; Kleerebezem, R.; Stams, A. J. M.; Kuenen, J. G.; Muyzer, G. Competition and coexistence of sulfate-reducing bacteria, acetogens and methanogens in a lab-scale anaerobic bioreactor as affected by changing substrate to sulfate ratio. Appl. Microbiol. Biotechnol. 2008, 78 (6), 1045-1055.

(38) Pareek, S.; Azuma, J. I.; Shimizu, Y.; Matsui, S. Hydrolysis of newspaper polysaccharides under sulfate reducing and methane producing conditions. Biodegradation. 2000, 11 (4), 229-237.

(39) Demoling, L. A.; Baath, E.; Greve, G.; Wouterse, M.; Schmitt, $\mathrm{H}$. Effects of sulfamethoxazole on soil microbial communities after adding substrate. Soil. Biol. Biochem. 2009, 41 (4), 840-848.

(40) Bundschuh, M.; Hann, T.; Gessner, M. O.; Schulz, R. Antibiotics as a chemical stressor affecting an aquatic decomposerdetritivore system. Environ. Toxicol. Chem. 2009, 28 (1), 197-203.

(41) Cooke, W. B. Fungi associated with the activated-sludge process of sewage treatment at the Lebanon, Ohio, sewage-treatment plant. Ohio. J. Sci. 1970, 70 (3), 129-146.

(42) Yiannakopoulou, T. V. An ecosystem analysis of the activated sludge microbial community. J. Environ. Sci. Health. 2010, 45 (5), $587-602$. 